Bundesgesundheitsbl - Gesundheitsforsch Gesundheitsschutz 2008 $\cdot 51: 994-1004$ DOI 10.1007/s00103-008-0627-1 Online publiziert: 7. September 2008 (c) Springer Medizin Verlag 2008

\author{
A. M. Wobus ${ }^{1} \cdot$ P. Löser ${ }^{2}$ \\ ${ }^{1}$ Leibniz-Institut für Pflanzengenetik und Kulturpflanzenforschung (IPK) Gatersleben, \\ BRD .2 Robert Koch-Institut, Berlin, BRD
}

\title{
Humane embryonale Stammzellen im Kontext internationaler Forschungs- aktivitäten
}

(ESchG) von 1991 verboten ist. Voraussetzung für Import und Verwendung von hES-Zellen nach dem StZG ist eine Genehmigung durch das Robert Koch-Institut (RKI). Im Rahmen des Genehmigungsverfahrens prüft die Zentrale EthikKommission für Stammzellenforschung (ZES), ob das Forschungsprojekt mit hESZellen den Vorgaben des $\S_{5}$ StZG entspricht.

Der folgende Beitrag zeigt auf, dass sich die hES-Zell-Forschung zu einem eigenständigen und expandierenden Forschungszweig entwickelt hat. Internationale Aktivitäten zur Standardisierung der Kultur und Aufbewahrung (Banking) von hES-Zellen, Bestrebungen zur Harmonisierung ethischer Standards bei der Arbeit mit diesen Zellen sowie bereits heute in der Entwicklung befindliche Anwendungen von hES-Zellen werden vorgestellt.

\section{Etablierung von pluripotenten humanen ES-Zell-Linien}

Ausgangsmaterial für die Etablierung humaner ES-Zell-Linien sind nach künstlicher Befruchtung (In-vitro-Fertilisation, IVF) gewonnene, frühe extrakorporale Embryonen, die nicht mehr für die Herbeiführung einer Schwangerschaft genutzt und in der Regel verworfen werden (sogenannte „überzählige“ Embryonen). Zur
Etablierung von hES-Zell-Linien werden die undifferenzierten embryonalen Zellen aus dem Inneren der Blastozyste (dem frühembryonalen „Blasenkeim“) isoliert und in Kulturmedium überführt [1]. Aus diesen Zellen werden hES-Zell-Linien etabliert, über längere Zeiträume kultiviert, kryokonserviert und anhand spezifischer Marker charakterisiert.

Da die Gewinnung von hES-Zell-Linien mit der Zerstörung von menschlichen Embryonen verbunden ist, wurden in den vergangenen Jahren alternative Verfahren für die Etablierung von hES-Zell-Linien entwickelt, die in der Perspektive die Zerstörung von Embryonen umgehen sollen bzw. unter Nutzung von Embryonen erfolgen, die bereits aus sich heraus keine oder verminderte Entwicklungschancen haben. Diese Verfahren haben zum Teil bereits zu neuen hES-Zell-Linien geführt [3]. Eine der Methoden beruht auf der Blastomeren-Biopsie, die im Rahmen von IVF-Behandlungen in vielen Ländern bei der Präimplantationsdiagnostik (PID) angewandt wird. Ziel ist es, aus einer Blastomere des Embryos eine hES-Zell-Linie herzustellen, ohne dabei das Entwicklungspotenzial des betreffenden Embryos zu beeinträchtigen. Dies ist bei der PID, die an einzelnen Zellen des sich entwickelnden Embryos im 8-16-Zell-Stadium durchgeführt wird, gängige Praxis. 2008 wurde erstmals über die erfolgreiche Ge- 
winnung von $5 \mathrm{hES}$-Zell-Linien aus Blastomeren berichtet, ohne dass dabei der Embryo zerstört wurde [4]. Ob diese Methode jedoch künftig von praktischer Relevanz für die Herstellung humaner ESZell-Linien sein wird, ist angesichts des bestehenden Risikos einer möglichen Schädigung des Embryos fraglich.

Als ethisch vertretbar wird in der Literatur auch die Möglichkeit diskutiert, Embryonen, die aufgrund des Vorliegens schwerster chromosomaler Anomalien geringe Entwicklungschance haben, zur Herstellung neuer hES-Zell-Linien zu nutzen. Es ist beispielsweise möglich, aus Embryonen mit chromosomalen Schädigungen genetisch intakte hES-Zell-Linien zu etablieren, wie kürzlich mit der Herstellung von 14 genetisch normalen Linien aus 61 Embryonen mit schweren chromosomalen Anomalien gezeigt wurde [5, 6]. Das Vorliegen eines normalen Karyotyps in einer hES-Zell-Linie, die aus einem genetisch geschädigten Embryo stammt, wird durch Mosaikbildung im frühen Embryo und Dominanz des normalen Karyotyps in der etablierten hESZell-Linie erklärt. Dieser Befund, der bereits zuvor beobachtet worden war [7], unterstreicht die Möglichkeit, aus Embryonen mit Chromosomenschäden (die für die Herbeiführung einer Schwangerschaft nicht infrage kommen) hES-Zell-Linien zu gewinnen. Auch die Etablierung von hES-Zellen aus sog. arretierten, natürlicherweise nicht mehr entwicklungsfähigen Embryonen wurde kürzlich beschrieben [8]. Diese Option war bereits als ethisch akzeptable Alternative zur Etablierung von hES-Zellen aus überzähligen Embryonen vorgeschlagen worden [9].

Die PID wird im Rahmen von IVF-Behandlungen zur vorgeburtlichen Diagnostik auf schwere Erbkrankheiten in zahlreichen Ländern eingesetzt, ist jedoch in Deutschland nicht statthaft. Im Ausland wurden aus Embryonen, die nach Feststellung eines schweren erblichen Defektes nicht mehr für die Herbeiführung einer Schwangerschaft genutzt und für die Forschung gespendet worden waren, mittlerweile zahlreiche hES-Zell-Linien mit definierten genetischen Defekten gewonnen, die als Zellmodelle für die Untersuchung der Pathogenese schwerer Erbkrankheiten

Tabelle 1

Humane embryonale Stammzell-Linien, die im Allgemeinen nach PID aus Embryonen abgeleitet wurden und genetische Veränderungen tragen, die mit menschlichen Erbkrankheiten in Zusammenhang stehen

\begin{tabular}{|c|c|c|}
\hline Krankheit & $\begin{array}{l}\text { Zahl der publizierten } \\
\text { hES-Zell-Linien }\end{array}$ & $\begin{array}{l}\text { Jahr(e) der } \\
\text { Veröffentlichung }\end{array}$ \\
\hline Chorea Huntington & 6 & 2005-2007 \\
\hline Fragiles X-Syndrom & 6 & $2005-2008$ \\
\hline Neurofibromatose, Typ 1 & 6 & 2005 \\
\hline Zystische Fibrose & 6 & $2005-2008$ \\
\hline Myotone muskuläre Dystrophie & 4 & 2005,2008 \\
\hline Thalassämie ( $\beta$-Locus) & 4 & 2005,2006 \\
\hline Muskeldystrophie, Typ Duchenne & 3 & 2005,2006 \\
\hline Marfan-Syndrom & 2 & 2006,2007 \\
\hline Muskeldystrophie, Typ Emery-Dreifuss & 2 & 2006 \\
\hline Adrenoleukodystrophie & 1 & 2005 \\
\hline Beta-Globin-Mutation IVS & 1 & 2006 \\
\hline Fanconi-Anämie & 1 & 2005 \\
\hline $\begin{array}{l}\text { Fazioskapulohumerale } \\
\text { Muskeldystrophie (FSHD) }\end{array}$ & 1 & 2007 \\
\hline Hämophilie A & 1 & 2008 \\
\hline Muskeldystrophie, Typ Becker & 1 & 2005 \\
\hline Okularer Albinismus & 1 & 2006 \\
\hline Primäre Torsionsdystonie (DYT1) & 1 & 2006 \\
\hline Sichelzell-Anämie & 1 & 2006 \\
\hline Spinale muskuläre Atrophie & 1 & 2006 \\
\hline Spinocerebelläre Ataxie Typ 7 & 1 & 2007 \\
\hline Thalassämie ( $\alpha$-Locus) & 1 & 2008 \\
\hline Waardenburg-Syndrom & 1 & 2004 \\
\hline Insgesamt (22 Erkrankungen) & 52 Zell-Linien & 2004-2008 \\
\hline
\end{tabular}

dienen können [10]. Die potenzielle Bedeutung solcher Linien für die Grundlagenforschung wurde eindrücklich an einer aus einem PID-Embryo abgeleiteten hES-Zell-Linie dokumentiert, die eine Mutation im FRAXA-Lokus trägt (Fragiles X-Syndrom) [11]. Es konnte nachgewiesen werden, dass der für die Erkrankung ursächliche Verlust der Expression des FMR1-Gens differenzierungsabhängig erfolgt. Die zuvor beobachtete und ursprünglich als für die Ausprägung der Krankheit bestimmend angesehene Methylierung des FMR1-Promotors erfolgt demnach erst nach Inaktivierung des FMR1-Gens, also nach der Etablierung des pathologischen Zustands. Insgesamt existieren derzeit mindestens 52 solcher aus PID-Embryonen gewonnenen hESZell-Linien (• Tabelle 1).

Die Einfuhr und Verwendung von hES-Zell-Linien, die nach PID gewonnen wurden, ist auch nach der Novellierung des StZG in Deutschland nicht zulässig. Die Nutzung solcher krankheitsspezifischen hES-Zell-Linien für die humangenetische Forschung ist in Deutschland folglich nicht möglich. Dagegen könnten genetisch modifizierte hES-Zell-Linien eingesetzt werden, wenn sie durch Veränderung bereits bestehender hES-Zell-Linien, die den Bedingungen des StZG entsprechen, gewonnen würden. So könnten Mutationen über das Verfahren der homologen Rekombination in hES-Zellen eingeführt oder durch phänotypische Ef- 
fekte durch RNAi-Technologien induziert werden [12]. Allerdings ist die homologe Rekombination an hES-Zellen bisher nur in wenigen Fällen erfolgreich eingesetzt worden [13].

Die Forschung an hES-Zellen wird in der Regel im Zusammenhang mit der Gewinnung transplantierbarer Zellen oder Gewebe für künftige Zellersatztherapien gesehen. Ein Defizit humaner embryonaler Stammzellen, die nach den oben genannten Methoden gewonnen werden, ist, dass die Zellen nicht immunkompatibel wären und aus ihnen hergestellte Transplantate als immunologisch fremd erkannt und gegebenenfalls abgestoßen würden. Dem Ziel der Gewinnung „patientenspezifischer" hES-Zell-Linien dient die somatische Kerntransfer-Technik (somatic cell nuclear transfer; SCNT, [14]) bzw. das „therapeutische Klonen“ [15], bei denen der Zellkern einer Körperzelle in eine entkernte Eizelle eingebracht wird. Der daraus entstehende nt-Embryo wird nach Entwicklung zur Blastozyste zur Gewinnung von nt-hES-Zellen verwendet. Die Herstellung von immunkompatiblen nt-hES-Zellen des Menschen ist bislang nicht gelungen, zudem ist die Erforschung dieses Verfahrens umstritten, insbesondere wegen des damit verbundenen hohen Bedarfs an menschlichen Eizellen; in Deutschland wäre die SCNT-Technik zur Herstellung von humanen nt-ES-Zellen ohnehin nach dem ESchG verboten. Die kürzlich publizierte erfolgreiche Klonierung menschlicher Embryonen, bei der aus den nt-Embryonen jedoch noch keine hES-Zell-Linien etabliert worden waren [16], hat das öffentliche Augenmerk jedoch wieder verstärkt auf diese Technik gerichtet.

Schon vor einigen Jahren war - als Alternative zum SCNT - der sogenannte „altered nuclear transfer" (ANT) ins Gespräch gebracht worden [17]. Beim ANT wird der Kern der somatischen Körperzelle, beispielsweise durch transiente Inaktivierung des Gens Cdx2, genetisch so verändert, dass eine Entwicklung des nach Kerntransfer entstehenden Embryos in vivo ausgeschlossen ist (z. B. infolge seiner Unfähigkeit, den Trophoblasten zu bilden und sich in den Uterus einzunisten). Aus solchen „depotenzierten“ Embryonen könnten jedoch in vitro ANT-hES-Zell-
Linien etabliert werden, in denen dann die Cdx2-Funktion wieder hergestellt würde. Die prinzipielle Funktionsfähigkeit dieses Verfahrens ist im Maussystem bereits gezeigt worden [18].

Die bisher genannten Verfahren stehen im Zusammenhang mit einer Zerstörung von menschlichen Embryonen, die (mit Ausnahme von nt-Embryonen) durch Verschmelzung von Ei- und Samenzelle entstanden sind. Dies trifft nicht für parthenogenetisch erzeugte Embryonen zu, aus denen ebenfalls hES-Zellen gewonnen werden können. Bei der Parthenogenese durchlaufen Eizellen eine Aktivierung durch chemische Substanzen oder elektrische Impulse, was zu einer artifiziell stimulierten Weiterentwicklung der Eizelle ohne vorherige Befruchtung führt. Erste parthenogenetisch hergestellte pluripotente Zell-Linien des Menschen (phESZell-Linien) sind jüngst beschrieben worden: dabei hatten 5 von 6 phES-ZellLinien einen normalen Karyotyp und zeigten während früher Differenzierungsprozesse ähnliche Eigenschaften wie hESZellen aus IVF-Embryonen [19]. Auch die bereits 2004 beschriebene, angeblich durch Kerntransfer erzeugte Linie „nthES-1“, erwies sich im Nachhinein als parthenogenetisch induzierte phES-Zell-Linie [20]. phES-Zell-Linien sind mit der Spenderin der Eizelle immunkompatibel, und demzufolge wären die aus ihnen abgeleiteten Zellen und Gewebe nach Transplantation in die Eizellspenderin voraussichtlich immunverträglich.

Eine vollkommen neue und alternative Methode zur Gewinnung von pluripotenten Zellen stellt die Strategie der induzierten Pluripotenz dar. Bereits 2006 war gezeigt worden, dass durch Transfer von 4 Genen, deren Produkte mit Pluripotenz assoziiert sind (Oct4, Sox2, c-myc, Klf4), aus Mausfibroblasten sogenannte „induzierte pluripotente Stammzellen“ (iPSZellen) erzeugt werden konnten [21]. Die iPS-Zellen zeigten große Ähnlichkeit mit murinen ES-Zellen, die auf konventionellem Wege entstanden sind. Nach Bestätigung der Befunde durch 3 unabhängige Arbeitsgruppen konnten durch dieses Verfahren Ende 2007 auch humane iPSZellen gewonnen werden [22, 23]. Inzwischen wurden in verschiedenen Labors aus unterschiedlichen somatischen Zellen humane iPS-Zell-Linien hergestellt und teilweise charakterisiert. Nachteile von iPS-Zellen im Vergleich zu ES-Zellen bestehen - neben ihrer noch unzureichenden Charakterisierung - gegenwärtig darin, dass ihre Herstellung derzeit den Transfer von Onkogenen in das zelluläre Genom erfordert und dass die Reprogrammierung noch an die Verwendung viraler Vektoren gebunden ist. Beides kann Ursache für die Entstehung von Tumoren aus iPS-Zellen sein [Übersicht in 24, 25].

\section{Standardisierung der Kultur, Verwendung und Aufbewahrung (Banking) von hES-Zellen}

\section{Charakterisierung von hES-Zell- Linien}

Seit der ersten Etablierung von hES-ZellLinien [1] wurden weltweit in zahlreichen Laboratorien neue Linien etabliert [2], die unter variablen Kulturbedingungen, in individuell hergestellten Medien und zunächst unter empirisch ermittelten Bedingungen kultiviert wurden, sodass die verfügbaren Linien in wichtigen Eigenschaften Unterschiede aufwiesen. Vor diesem Hintergrund - und um das verfügbare Zellmaterial unter vergleichbaren Bedingungen zu charakterisieren - rief das Internationale Stammzellforum (ISCF, http://www.stemcellforum.org.uk) im Jahr 2003 eine Internationale StammzellInitiative (ISCI) ins Leben, die eine vergleichende Charakterisierung von hESZell-Linien koordinierte [26]. Im Rahmen einer ersten Studie (ISCI-1) wurden 59 unabhängig voneinander etablierte hESZell-Linien (darunter 18 Linien des NIHRegisters) aus 17 Laboratorien in 11 Ländern hinsichtlich ES-Zell-spezifischer Parameter (Zell-Oberflächenantigene, Genexpressionsmuster, epigenetischer Status, Differenzierungsfähigkeit nach Injektion in immundefiziente Mäuse) charakterisiert.

Alle untersuchten Linien wiesen eindeutige Merkmale pluripotenter embryonaler Stammzellen auf, auch wenn die Studie eine gewisse Variabilität zwischen den Linien erkennen ließ. Als eindeutige Pluripotenzmarker wurden die Zelloberflächenantigene SSEA-3 und -4, TRA-16o, TRA-1-81, TRA-2-54, CD9, GCTM2 und GCTM343 identifiziert, während an- 
dere Marker, wie SSEA-1, $\mathrm{A}_{2} \mathrm{~B}_{5}, \mathrm{CD}_{5} 6$ (NCAM), GD2 und 3 nicht in allen Linien gleichmäßig stark exprimiert waren. Die Analyse der Expression einer Reihe von Genen und der Vergleich mit der Expression des besonders stark mit Pluripotenz assoziierten Gens nanog ergab übereinstimmend, dass die Gene TDFG, POU $5 \mathrm{~F}_{1}$ (Oct3/4), GABRB3, GDF3 und $\mathrm{DNMT}_{3} \mathrm{~B}$ in allen untersuchten Linien hoch exprimiert waren und ihre Expression stark mit dem pluripotenten Zustand von hES-Zellen assoziiert war. 14 weitere Gene, die u. a. auch in aus hES-Zellen abgeleiteten, bereits in Differenzierung befindlichen Zellen exprimiert werden (FGF4, GAL, LEFTB, IFITM1, NODAL, TERT, UTF1, FOXD 3, LIN28, GRB7, PODXL, CD9 und BRIX), wiesen hinsichtlich ihrer Expression eine schwächere Korrelation mit der Expression des nanog-Gens und eine z. T. stärkere Variabilität auf.

Im Rahmen der Analyse des epigenetischen Status der hES-Zell-Linien wurde erneut sichtbar, dass hES-Zell-Linien deutliche epigenetische Varianzen aufweisen können. In der genannten Studie wurden 10 Gene untersucht, die mit genomischem Imprinting korreliert sind. Genomisches Imprinting führt in der Regel zur Inaktivierung von Genen mit der Folge, dass die betreffenden Gene entweder vom maternal oder paternal erworbenen Allel (monoallelisch), jedoch nicht von beiden Allelen (biallelisch), exprimiert werden. In hES-Zellen scheint bei einigen Genen ein Verlust des Imprintings (loss of imprinting, LOI) zu erfolgen, der eine teils große Variabilität zwischen den Linien hinsichtlich der mono- oder biallelischen Expression der Gene zur Folge hat. Dies betrifft beispielsweise das Gen für den Insulin-ähnlichen Wachstumsfaktor 2 (IGF2). Ferner bestätigte die Studie, dass bezüglich des Status der X-Chromosomen-Inaktivierung große Unterschiede zwischen „weiblichen“ hES-Zell-Linien bestehen, was z. T. auf suboptimale Kulturbedingungen zurückgeführt und auch durch Ergebnisse anderer Studien belegt wurde $[27,28,29]$. Normalerweise erfolgt die für die Entwicklung notwendige Inaktivierung des zweiten X-Chromosoms durch Bindung einer spezifischen RNA (X inactive specific transcript, XIST). Die Fähigkeit zur Expression des XIST-Gens

Bundesgesundheitsbl - Gesundheitsforsch - Gesundheitsschutz 2008 · 51:994-1004 DOI 10.1007/s00103-008-0627-1

(c) Springer Medizin Verlag 2008

\section{A. M. Wobus · P. Löser}

\section{Humane embryonale Stammzellen im Kontext internationaler Forschungsaktivitäten}

\section{Zusammenfassung}

Forschung an pluripotenten humanen embryonalen Stamm (hES)-Zellen ist ein rasch wachsendes Wissenschaftsgebiet. Aufgrund der Herkunft des Zellmaterials aus frühen menschlichen Embryonen wurden alternative Verfahren zur Gewinnung von pluripotenten Zellen entwickelt. Der Beitrag stellt einige dieser Strategien vor und behandelt darüber hinaus die internationalen Bestrebungen, einheitliche Standards für die Kultivierung, Charakterisierung und Lagerung von $\mathrm{hES}$-Zellen zu etablieren. Ferner werden die weltweiten Tendenzen zur Bildung von Netzwerken auf dem Gebiet der Stammzellforschung sowie Bestrebungen zur Harmonisierung ethischer Standards bei der Arbeit mit hESZellen aufgezeigt. Schließlich werden Perspektiven der Verwendung von hES-Zellen in der pharmakologisch-toxikologischen Forschung sowie jüngste Ergebnisse tierexperimenteller Studien mit hES-Zellen diskutiert.

\section{Schlüsselwörter}

Humane embryonale Stammzellen . hES-Zellen · Charakterisierung ·

Forschungsnetzwerke . Anwendung

\section{Human embryonic stem cells within the context of international research activity}

\section{Abstract}

Research involving pluripotent human embryonic stem cells ( $\mathrm{hESCs}$ ) is a rapidly growing field of science. Since hESCs originate from early human embryos, alternative methods for producing pluripotent cells have been developed. This article introduces some of those strategies and, in addition, covers international efforts to establish consistent international standards for cultivation, characterization and preservation of hESCs. Furthermore, global trends to form networks in the field of stem cell research as well as endeavors to harmonize ethical standards for $\mathrm{hESC}$ research are presented. Finally, potential applications of hESCs in the field of pharmacology/toxicology are discussed as well as recent results of animal studies using $\mathrm{hESC}$.

\section{Keywords}

Human embryonic stem cells $\cdot h E S C s$. characterization - research networks . application 
und damit zur Inaktivierung des zweiten $\mathrm{X}$-Chromosoms differiert jedoch zwischen verschiedenen hES-Zellen erheblich, bis hin zum Verlust der Fähigkeit, XIST überhaupt produzieren zu können. In diesem Zusammenhang wurde kürzlich gezeigt, dass die Fähigkeit zur Expression von XIST offenbar mit dem Differenzierungspotenzial der entsprechenden hES-Zell-Linie korreliert sein kann [28].

Die Differenzierungskapazität von hES-Zell-Linien kann bestimmt werden, indem undifferenzierte hES-Zellen in immundefiziente Mäuse injiziert und die sich entwickelnden Teratome bezüglich des Auftretens bestimmter Zell- und Gewebetypen histologisch untersucht werden. Auch in der ISCI-1-Studie wurden 15 Linien hinsichtlich der Entwicklung von Geweben aller 3 Keimblätter untersucht. Es wurden frühe ektodermale, mesodermale, endodermale und vor allem unreife neurale Gewebe nachgewiesen. Dagegen wurden undifferenzierte (embryonalen Karzinomzellen ähnliche) Zelltypen nur in 3 der untersuchten Teratome beobachtet. In-vitro-Differenzierungen in spezifische Zelltypen wurden hier zwar nicht systematisch analysiert, es zeigten sich jedoch Unterschiede im In-vitro-Differenzierungsverhalten einzelner hESZell-Linien, was frühere Beobachtungen zum unterschiedlichen Differenzierungspotenzial von hES-Zellen bestätigt [30, 31]. Zudem ergab ein kürzlich publizierter Vergleich von $17 \mathrm{hES}$-Zell-Linien (HUES 1-17) [32] gravierende Unterschiede in der Differenzierungsfähigkeit zwischen den untersuchten Linien mit bis zu 100-fachen Unterschieden in der Expressionsrate von Genen, deren Produkte mit der Differenzierung in gewebespezifische Zelltypen assoziiert sind. Humane ES-Zell-Linien, die sich gut in Blutzellen differenzieren ließen, zeigten beispielsweise kaum neuronale Differenzierung, andere Linien differenzierten effizient vorzugsweise in pankreatische, andere in kardiale Zellen [33].

Wichtige Forschungsfelder an hESZellen sind weiterhin die Untersuchung der Mechanismen, die die Pluripotenz von ES-Zellen erhalten, die Analyse der Regulation Pluripotenz-assoziierter Signalwege sowie die Erforschung von Mechanismen, die der Auslösung von Diffe- renzierungsprozessen zugrunde liegen [34]. Grundlegende Regulationsmechanismen für die Aufrechterhaltung der Pluripotenz sind in murinen und humanen ES-Zellen unterschiedlich. So ist das Signalmolekül LIF nur in murinen ES-Zellen für die Beibehaltung des undifferenzierten Status erforderlich [35], nicht aber in humanen ES-Zellen [36]. Dagegen sind u. a. die Faktoren FGF-2 [37] und Activin A [38] entscheidend für die Aufrechterhaltung der Pluripotenz von hES-Zellen, während sie in murinen ES-Zellen Differenzierung auslösen. In diesem Zusammenhang ist erwähnenswert, dass kürzlich ein neuer Typ pluripotenter Stammzellen aus dem Epiblasten früher Mausembryonen isoliert und kultiviert wurde. Diese pluripotenten Zellen weisen Eigenschaften auf, wie sie von humanen, nicht aber von murinen ES-Zellen bekannt sind, und werden als möglicher analoger Zelltyp zu hES-Zellen diskutiert [39].

Während die Aufrechterhaltung von Pluripotenz in den Stammzellen unterschiedlicher Spezies offenbar auf verschiedenen Wegen gewährleistet wird, bestehen bezüglich der Differenzierungsinduktion in Zellderivate der verschiedenen Keimblätter kaum prinzipielle Unterschiede in den Differenzierungsfaktoren zwischen mES- und hES-Zellen. Aufgrund der Fülle der Daten kann hier nur auf die Literatur verwiesen werden (Übersichtsarbeiten in $[40,41])$.

\section{Entwicklung von standardisierten Kulturverfahren}

Grundlage für eine bessere Vergleichbarkeit von Resultaten der hES-Zell-Forschung ist unter anderem die Kultivierung von hES-Zell-Linien unter standardisierten Bedingungen. Um dies zu erreichen, müssen langfristig jene Komponenten von der Kultivierung der hES-Zellen ausgeschlossen werden, die eine Standardisierung der Kulturen nicht zulassen. Dies betrifft in erster Linie murine feeder-Zellen, auf denen hES-Zellen in der Regel kultiviert werden, aber auch nicht-standardisierbare tierische Komponenten, wie beispielsweise fötales Kälberserum. Darüber hinaus wäre es im Hinblick auf eine künftige therapeutische Nutzung von De- rivaten aus hES-Zellen wünschenswert, tierische Komponenten insgesamt bei der Kultivierung von hES-Zellen zu vermeiden, diese also vollkommen xenogenfrei durchzuführen. hES-Zellen werden bereits heute in verschiedenen Labors entweder mit kommerziell verfügbaren humanen feeder-Zellen oder ohne feederZellen kultiviert. Als Medienzusätze (und Serumersatz) kommen standardisierte Supplemente, wie serum replacement (SR), knockout serum replacement (KSR) oder extrazelluläre Matrix-Proteine, beispielsweise Collagen, Plasmin oder Matrigel, zum Einsatz (Übersicht in [42]). Allerdings enthalten SR, KSR und Matrigel noch Komponenten tierischer Herkunft. Neben zahlreichen individuell hergestellten Kulturmedien sind inzwischen auch kommerziell verfügbare Medien im Einsatz, mit denen hES-Zellen in Abwesenheit von Serum und teils ohne feeder-Zellen kultiviert werden können. Im Rahmen der Standardisierungsoffensive (ISCI-2 Phase des ISCF) werden 3 dieser Medien (mTeSR 1, STEMPRO ${ }^{\circledR}$ hESC SFM und HEScGRO) zusätzlich zu einigen individuell hergestellten Kulturmedien systematisch auf ihre Eignung für die Kultivierung von hES-Zellen untersucht. Zudem wurde jüngst die Gewinnung und Kultivierung von hES-Zellen auch unter Nutzung vollständig synthetischer Medien beschrieben [43].

Nach wie vor wird intensiv an der Entwicklung von hES-Zell-Linien gearbeitet, die sich für die Herstellung von Geweben für die klinische Anwendung eignen. Voraussetzung dafür ist, dass die hES-Zell-Linien unter standardisierten Bedingungen und in "guter Herstellungspraxis" (good manufacturing practice, GMP) gewonnen wurden, wobei die Verwendung tierischer Zellen oder Proteine im gesamten Kultivierungsprozess ausgeschlossen sein sollte. Kürzlich wurden 6 derartige, GMPzertifizierte hES-Zell-Linien der Firma ES Cell International (Singapur) publiziert, die 2006 ohne wesentliche tierische Komponenten etabliert wurden und in Kürze für Forschungszwecke verfügbar sein werden [44]. Über die Existenz weiterer GMP-gerechter hES-Zell-Linien wurde von den Firmen Cellartis (Göteborg, Schweden), NovoCell (San Diego, CA, USA) und Advanced Cell Technology 
(ACT, Los Angeles, CA, USA) sowie vom Korean Stem Cell Research Center (Seoul, Südkorea) berichtet.

Allerdings müssen, zusätzlich zur standardisierten Kultur von hES-Zellen, weitere Aspekte berücksichtigt werden, die gegebenenfalls Auswirkungen auf die Vergleichbarkeit von Forschungsergebnissen haben können: Dies betrifft beispielsweise die Tatsache, dass sich Embryonen während der präimplantativen Entwicklung hinsichtlich des Status der DNA-Methylierung und Histonmodifikationen unterscheiden, was Auswirkungen auf die Regulation bestimmter Pluripotenz-assoziierter Gene in den aus ihnen abgeleiteten hES-Zellen haben kann. Dies kann in der Folge zu Unterschieden - auch im Differenzierungsverhalten - zwischen einzelnen hES-Zell-Linien führen [33, 45]. Zudem ist bekannt, dass sich in hES-Zellen während ihrer Langzeitkultivierung genetische und epigenetische Veränderungen vollziehen können. Voraussetzung für die künftige Anwendung von hES-Zellen, sei es in der Zellersatztherapie oder in der Arzneimittelentwicklung, ist, dass diese Parameter stabil bleiben, sich also weder chromosomale Aberrationen, Mutationen oder epigenetische Veränderungen in Abhängigkeit von den Kulturbedingungen entwickeln [45]. Ein stetiges Monitoring der verwendeten Linien hinsichtlich dieser Parameter ist daher unabdingbar.

\section{Internationale Aktivitäten zur Standardisierung und Harmoni- sierung der hES-Zellforschung}

Eine Übersicht über die internationale Stammzellforschung registriert weltweit mindestens 120 Forschungszentren, die Forschung an humanen ES-Zellen durchführen (http://www.mbbnet.umn.edu/scmap/scresearchmap.html). In den meisten Ländern, in denen Stammzellforschung betrieben wird, wurden verschiedene nationale Netzwerke etabliert, so $u$. a. auch in Deutschland (z. B. Stammzellnetzwerk Nord-Rhein-Westfalen, http://www. stammzellen.nrw.de), in Großbritannien (UK National Stem Cell Network, UKSCNW, http://www.uknscn.org; Scottish Stem Cell Network, SSCN, http://www. sscn.co.uk), Australien (Australian Stem Cell Centre, ASCC, http://www.stemcell- centre.edu.au) oder Kanada (http://www. stemcellnetwork.ca). Daneben wurden länderübergreifende Organisationen geschaffen, wie beispielsweise das von Organisationen aus 11 skandinavischen und Ostsee-Anrainer-Staaten gegründete Netzwerk „ScanBalt BioRegion“ (http:// www.scanbalt.org).

Um die internationale Zusammenarbeit insbesondere auch auf dem Gebiet der humanen embryonalen Stammzellforschung zu fördern, wurde 2003 das Internationale Stammzell-Forum (International Stem Cell Forum, ISCF, http://www. stemcellforum.org) geschaffen. Ziel des ISCF ist es, die weltweiten Aktivitäten der Stammzellforschung zu unterstützen und zu koordinieren, die Etablierung und Kultivierung von Stammzellen (nach GLP, GMP) zu standardisieren und sich für eine internationale Harmonisierung ethischer Standards in der Forschung mit Stammzellen einzusetzen. Mitglieder des ISCF sind 21 internationale staatliche und private Förderorganisationen und Institutionen aus 19 Ländern, die auf dem Gebiet der Stammzellforschung arbeiten. Im Rahmen des ISCF wurden weitere Initiativen gegründet, wie die Internationale Stammzell-Initiative (ISCI), die Standards für die Etablierung, Charakterisierung und Erhaltung von hES-Zell-Linien erarbeitet; die Internationale Stammzell-Bank Initiative (International Stem Cell Banking Initiative, ISCBI), die ein globales Netzwerk von Stammzell-Banken etabliert; die Ethics Working Party (EWP), die die ethischen Standards und Grundlagen ethischer Entscheidungen weltweit vergleichend referiert (http://www.stemgen. org), sowie eine Arbeitsgruppe zu den Rechten an geistigem Eigentum (Intellectual Property Rights), die sich mit der Patentierung von hES-Zell-Linien und unterschiedlichen rechtlichen Rahmenbedingungen in verschiedenen Ländern befasst.

Darüber hinaus sind weitere internationale Organisationen auf dem Gebiet der Stammzellforschung aktiv. Die Internationale Gesellschaft für Stammzellforschung (International Society for Stem Cell Research, ISSCR, http://www.isscr. org) ist eine internationale Vereinigung von Stammzellforschern mit weltweit über 2000 Mitgliedern. Die ISSCR führt jährlich internationale Tagungen durch, die bisher auf allen Kontinenten, in Amerika, Australien, Asien und Europa, stattfanden bzw. geplant sind [z. B. 2008: 4. ISSCR-Kongress in Philadelphia (USA), 2009 5. ISSCR-Kongress in Barcelona (Spanien)]. Die ISSCR hat ebenfalls Regeln für das Arbeiten mit humanen embryonalen Zellen (Guidelines for the Conduct of Human Embryonic Research, siehe http://www.isscr.org/guidelines) aufgestellt, in denen insbesondere ethische Regeln im Umgang mit menschlichen Embryonen formuliert sind. Die ISSCR koordiniert Arbeitsgruppen, die sich mit spezifischen Fragen der Stammzellforschung befassen, wie z. B. die „ISSCR Task Force on the Clinical Translation of Stem Cells" [Kooperationen u. a. mit Initiativen der European Science Foundation (ESF)]. Die ISSCR ist Mitglied im Internationalen Konsortium der Stammzell-Netzwerke (International Consortium of Stem Cell Networks, ICSCN, http://icscn.wordpress. com), sie kooperiert u. a. mit der Human Proteom Organisation (HUPO, http:// www.hupo.org) zur Erforschung des Proteoms von Stammzellen oder der Internationalen Organisation zur Forschung an Typ1-Diabetes (Juvenile Diabetes Research Foundation International, JDRF, http://www.jdrf.org).

Nationale und internationale Register, die die etablierten humanen ES-Zellen hinsichtlich Etablierung, Eigenschaften und Verfügbarkeit registrieren, werden von verschiedenen Organisationen und Initiativen geführt. Beispielhaft seien die Register der National Institutes of Health (NIH) der USA (http://stemcells.nih.gov), der Britischen Stammzellbank am Medical Research Council (UK Stem Cell Bank, http://www.ukstemcellbank.org), des Internationalen Stammzellforums (ISCF, http://www.stemcellforum.org/isci_project/the_registry.cfm) und der Europäischen Union (European Human Embryonic Stem Cell Registry (hESCReg; http:// www.hesreg.eu) genannt (• Tabelle 2).

Stammzellbanken wurden mittlerweile von verschiedenen internationalen Organisationen und Institutionen etabliert. Die Internationale Stammzell-Banking Initiative (International Stem Cell Banking Initiative, ISCBI; http://www.stemcellforum. org) des ISCF erarbeitet $u$. a. Richtlinien 
Tabelle 2

Derzeit existierende öffentliche hES-Zell-Register und Zellbanken, die hESZellen lagern. Die Register führen lediglich Zell-Linien auf, nennen deren Verfügbarkeit beim Hersteller und enthalten teilweise Daten zu den Eigenschaften der Zell-Linien. Von den Banken ist dagegen ein direkter Bezug der jeweils enthaltenen Linien möglich. Es ist zu beachten, dass einige Linien zugleich in mehreren Registern bzw. Banken enthalten sein können

\begin{tabular}{lcc}
$\begin{array}{l}\text { hES-Zell-Register, } \\
\text { hES-Zell-Bank }\end{array}$ & $\begin{array}{l}\text { Zahl der gelisteten } \\
\text { hES-Zell-Linien }\end{array}$ & $\begin{array}{l}\text { Zahl der verfügbaren } \\
\text { hES-Zell-Linien }\end{array}$ \\
\hline NIH Registry & 72 & 21 \\
\hline EU hESC registry & 206 & 114 \\
\hline ICSF Registry & 57 & 57 \\
\hline National Stem Cell Bank (USA) & 18 & 14 \\
\hline UK Stem Cell Bank & 55 & 8 \\
\hline Singapore Stem Cell Bank & 4 & 0 \\
\hline
\end{tabular}

zur Standardisierung und Datenerfassung für die Langzeit-Aufbewahrung (best practice for banking) von hES-Zell-Linien (Publikation ist für 2008 angekündigt). Die UK Stem Cell Bank des Medical Research Council (MRC) (http://www.ukstemcellbank.org), die derzeit 8 charakterisierte humane ES-Zell-Linien für weltweite Forschungsarbeiten zur Verfügung stellt und weitere ca. 50 Linien für die Aufnahme vorbereitet, hat einen hohen Standard bezüglich wissenschaftlicher und ethischer Parameter zur Einlagerung von hES-Zellen und damit Vorbildfunktion für viele Initiativen. Darüber hinaus entwickelt das Netzwerk der Human Embryonic Stem Cell Coordinators (hESCCO) GMP-Standards für die Gewinnung und Aufbewahrung von humanen ES-Zellen in Großbritannien [46]. In den USA wurde eine Nationale Stammzellbank (National Stem Cell Bank, NSCB, http://www.nationalstemcellbank.org/) etabliert, die derzeit 14 von 21 hES-Zell-Linien des NIH-Registers zur Verfügung stellt. Diese können für Forschungsarbeiten mit NIH-Mitteln eingesetzt und auch von deutschen Forschern genutzt werden. Die Firma Stemride International Limited (SIL, http://www. stemride.com), die mit dem Reproductive Genetics Institute in Chicago (USA) zusammenarbeitet, bietet derzeit über $150 \mathrm{hES}-Z$ ell-Linien, darunter mehr als 20 Linien mit spezifischen genetischen Defekten an (siehe $\bullet$ Tabelle 1). Weitere Stammzellbanken, die hES-Zellen enthalten, existieren bereits (wie beispielsweise die Bank des Singapore Stem Cell Consortium, http://www.sscc.a-star.edu.sg) oder befinden sich im Aufbau (wie beispielsweise am Stem Cell Research Center in Seoul, Korea, http://koreastemcellbank. org).

\section{Potenzielle Einsatzmöglichkeiten von $\mathrm{hES}$-Zellen}

\section{Einsatz von hES-Zellen in der Wirkstoff-Forschung (drug development) und zur In-vitro- Toxizitätstestung}

Der Einsatz von hES-Zellen in der Wirkstoff-Forschung und für die Entwicklung von Medikamenten und Toxizitätstests ist das aus heutiger Sicht anwendungsnächste Gebiet der hES-Zellforschung [47]. Grundlage für den Einsatz von hES-Zellen in diesen Bereichen ist, dass hES-Zellen in vitro in zahlreiche spezialisierte humane Zelltypen differenziert werden können. Aus pharmakologisch-toxikologischer Sicht interessante Zelltypen sind dabei insbesondere Leberparenchymzellen (Hepatozyten), Herzmuskelzellen (Kardiomyozyten) und verschiedene neuronale Zellen. Für die Differenzierung in diese Zellen wurden in den letzten Jahren spezifische Differenzierungsprotokolle etabliert und optimiert, sodass erwartet wird, dass in Kürze definierte humane Zellen in ausreichender Zahl zur Verfügung stehen werden, die die Eigenschaften der entsprechenden primären mensch- lichen Zellen aufweisen. An ihnen können zum einen spezifische Wirkungen von pharmakologisch aktiven Substanzen auf zellulärer Ebene im Hochdurchsatzverfahren untersucht werden (drug screening). Mithilfe der hES-Zell-Technologie können zum anderen niedermolekulare Substanzen (small molecules) identifiziert werden, die die Regeneration von Geweben aus Vorläuferzellen stimulieren [48]. Ganz wesentlich können hES-Zell-abgeleitete Zellen bereits in einer sehr frühen Phase der Medikamentenentwicklung zur Untersuchung humanspezifischer Toxizität genutzt werden. Dadurch würden entsprechende Substanzen - im Falle von Hinweisen auf unerwünschte Wirkungen - bereits in einem frühen Stadium der Medikamentenentwicklung von der weiteren Untersuchung ausgeschlossen. Der Vorteil der Nutzung von aus hES-Zellen gewonnenen Zellen gegenüber tierischen Primärzellen bestünde (neben der Einsparung von Versuchstieren) vor allem in der Humanspezifität des jeweiligen Testsystems, was - angesichts der Defizite hinsichtlich der Übertragbarkeit von Ergebnissen aus Versuchen mit tierischen Zellen auf den Menschen - einen erheblichen Fortschritt gegenüber den derzeit verfügbaren, z. B. auf primären tierischen Zellen basierenden Testsystemen darstellen würde. Vorteile von aus hES-Zellen abgeleiteten Zellen gegenüber humanen Primärzellen wären die faktisch unbegrenzte Verfügbarkeit, die hohe Reinheit sowie ihre gute Standardisierbarkeit. Zusätzlich ließen sich durch Nutzung spezialisierter Zellen, die aus hES-Zellen mit unterschiedlichen genetischen Eigenschaften differenziert werden, Pharmaka bereits im Vorfeld klinischer Studien im Hinblick auf pharmakotoxische Eigenschaften in Abhängigkeit von Genotyp oder ethnischer Herkunft des hES-Zellspenders in Zellkultur untersuchen.

Eine weitere Möglichkeit der Nutzung von hES-Zellen in der pharmakologischtoxikologischen Forschung besteht in der Untersuchung von humanspezifischer Entwicklungs- bzw. Embryotoxizität. Durch Untersuchungen an sich differenzierenden Zellen könnten bestimmte Aussagen darüber getroffen werden, ob oder inwieweit Pharmaka oder Umweltfaktoren die Differenzierung spezifischer 
Zelltypen des Menschen beeinträchtigen. Solche Untersuchungen werden bereits heute unter Verwendung von ES-Zellen der Maus im sogenannten „Embryonalen Stammzelltest“" (EST) zur Bestimmung des embryotoxischen Potenzials von Testsubstanzen durchgeführt. Der EST mit Mauszellen ist jedoch mit dem Nachteil verbunden, dass humanspezifische Entwicklungstoxizität (wie sie beispielsweise im Fall von Contergan bestand) nicht zwingend im Mausmodell erfasst würde. Ziel ist es nun, entsprechende Testverfahren auf der Basis von hES-Zellen zu entwickeln. Dabei steht zunächst die Ermittlung geeigneter Endpunkte für die Bestimmung der toxischen Wirkung von ausgewählten Substanzen im Zentrum der Arbeiten [49].

Ein Schwerpunkt der Bestrebungen bei der Herstellung pharmakologisch-toxikologisch verwendbarer Zellen aus hES-Zellen ist die Herstellung von funktionalen menschlichen Hepatozyten [50]. Diese Zellen werden u. a. benötigt, um den Leberstoffwechsel sowie pharmakokinetische Eigenschaften neuer Medikamente zu untersuchen. Dabei ist das Vorhandensein induzierbarer Arzneimittel-metabolisierender, -konjugierender und -transportierender Enzyme in den Leberzellen wesentlich, um zu belastbaren Aussagen über die Eigenschaften der untersuchten Substanzen in der menschlichen Leber zu gelangen. Etablierte Leberzell-Linien (wie z. B. die vielfach verwendete HepatomZell-Linie HepG2 und ihre Derivate) wie auch Leberzellen aus anderen Quellen (z. B. aus mesenchymalen Stammzellen differenzierte Hepatozyten) produzieren diese Enzyme jedoch nur in geringem Maße. Die fehlende Verfügbarkeit geeigneter humanspezifischer Leberzell-basierter Testsysteme wird als eine Ursache dafür angesehen, dass pharmakokinetische und toxikologische Probleme, die mit der Metabolisierung von Arzneimitteln in der Leber zusammenhängen und Ursache für unerwartete, teils fatale Nebenwirkungen von Medikamenten sein können, häufig erst in späten Phasen der Arzneimittelentwicklung erkannt werden. Derzeit existieren verschiedene Protokolle für die In-vitro-Differenzierung von hES-Zellen zu Hepatozyten, wobei die In-vivo-Differenzierung von Leberzellen während der menschlichen Entwicklung simuliert wird. Vielversprechende Ergebnisse auf diesem Gebiet wurden u. a. unter Mitwirkung der in der hES-Zell-Forschung engagierten Firmen Cellartis, Geron und ACT $[51,52,53]$ erzielt. Dass auch große Unternehmen der Pharmaindustrie ihr Augenmerk zunehmend auf diesen speziellen Aspekt der Forschung mit hES-Zellen richten, wird unter anderem in der Gründung des Konsortiums „Stem Cells for Safer Medicine" ersichtlich, an dem neben dem britischen Medical Research Council (MRC) u. a. 3 große europäische Pharmafirmen (Roche, AstraZeneca, GlaxoSmithKline) beteiligt sind. Ziel dieses Konsortiums ist es, Verfahren für die Herstellung von Hepatozyten für die Toxizitätstestung aus hES-Zellen zu entwickeln.

Weltweit sind zahlreiche Firmen auf verschiedenen Gebieten der hES-ZellForschung tätig (• Tabelle 3). Angesichts des absehbaren Potenzials von hES-Zellen für die Medikamentenentwicklung ist auch in der Haltung der Pharmaindustrie eine Trendwende zu konstatieren: Während in der Vergangenheit von großen Pharmaunternehmen eher Zurückhaltung in Bezug auf den Einsatz von hES-Zellen zu beobachten war, bekennen sich inzwischen weltweit führende Pharmafirmen öffentlich zur Forschung mit hES-Zellen und haben entsprechende Entwicklungsund Kooperationsverträge mit spezialisierten Institutionen abgeschlossen bzw. eigene Forschungsprogramme aufgelegt. Der in der jüngsten Debatte um die Novellierung des StZG vermittelte Eindruck, die pharmazeutische Industrie habe kein Interesse an der Forschung mit hES-Zellen, mag für deutsche Firmen oder deren Tätigkeit im Inland gelten, ist jedoch international gesehen nicht zutreffend.

\section{Transplantation von aus $\mathrm{hES}$-Zellen} entwickelten Spenderzellen in Tiermodelle humaner Erkrankungen

Die Forschung an hES-Zellen war und ist mit der Hoffnung auf Zelltherapien für bisher anderweitig nicht heilbare degenerative Erkrankungen, wie Diabetes mellitus oder Morbus Parkinson, verbunden. Die Entwicklung eines spezifischen Medikamentes bis zur Marktreife kann bis zu 15 Jahre in Anspruch nehmen. Auch die
Entwicklung anderer therapeutischer Ansätze von der Grundlagenforschung bis zur Anwendung benötigt häufig lange Zeiträume. Insofern sind Erwartungen, dass nur 10 Jahre nach Begründung einer neuen Forschungsrichtung bereits Therapien, die auf dieser Forschung beruhen, verfügbar sein sollten, im Allgemeinen nicht gerechtfertigt. Zwar gibt es derzeit noch keine klinische Studie unter Nutzung von aus hES-Zellen abgeleitetem Material, jedoch wurde über die Vorbereitung entsprechender Studien mehrfach berichtet, insbesondere von der Firma Geron, die seit 2005 wiederholt über die Planung einer Studie zur Therapie von akuten Verletzungen des Rückenmarks informiert hat. Allerdings wurde die Studie kürzlich von der zuständigen US-Behörde (Federal Drug Agency, FDA) gestoppt, ohne dass die Gründe dafür derzeit öffentlich bekannt sind [54]. In den USA bestehen aber weitere intensive Bemühungen, die konkreten Zulassungsvoraussetzungen für hES-Zell-basierte Therapien zu klären [55]. So wurde berichtet, dass auch die Firma Advanced Cell Technology (ACT) ein Zulassungsvorgespräch für eine klinische Studie (pre-IND-meeting) mit der FDA über die Nutzung einer hES-Zell-basierten Zelltherapie für den Ersatz des retinalen Pigmentepithels zur Therapie der altersbedingten Makuladegeneration geführt hat [56]. Sollten die Voraussetzungen für klinische Studien geklärt sein, so werden diese, wie üblich, Untersuchungen zur klinischen Verträglichkeit und Unbedenklichkeit enthalten, wobei das Augenmerk im Falle von hESZell-abgeleitetem Material besonders auf der Vermeidung von Tumorbildung liegt.

Insgesamt befindet sich die Forschung an hES-Zellen für Zwecke der regenerativen Medizin gegenwärtig noch im Stadium der Grundlagenforschung, wobei bereits heute in Tiermodellen verschiedener menschlicher Erkrankungen der proofof-concept für einen potenziellen Einsatz hES-Zell-basierter Therapien gezeigt wurde. Allerdings sind vor einem klinischen Einsatz von Zellen oder Geweben, die aus hES-Zellen abgeleitet wurden, 2 wesentliche Probleme zu lösen:

1) Vor jeder möglichen Transplantation muss so weit wie möglich sichergestellt sein, dass das Transplantat keine „kon- 
Tabelle 3

\section{Beispiele für Firmen, die auf dem Gebiet der Forschung mit hES-Zellen tätig sind}

\begin{tabular}{ll}
\hline Firmenname & Standort \\
\hline Advanced Cell & Los Angeles, USA \\
Technologies (ACT) &
\end{tabular}

\section{Forschungsaktivitäten mit hES-Zellen}

Verfügt über mehrere hES-Zell-Linien

Differenzierung u. a. in Retina-Epithelium sowie Gefäßvorläufer für therapeutische

Anwendungen

Differenzierung zu Hepatozyten für klinische und pharmakologisch-toxikologische Applika-

tionen

\begin{tabular}{|c|c|c|}
\hline Astra Seneca & London, GB & $\begin{array}{l}\text { Entwicklung von Hepatozyten/Kardiomyozyten für Studien zu Wirkung, Metabolismus und } \\
\text { Toxizität von Arzneimitteln } \\
\text { Kooperation mit externen Partnern }\end{array}$ \\
\hline Axordia & Sheffield, GB & $\begin{array}{l}\text { Gewinnung von GMP-konformen hES-Zell-Linien } \\
\text { Aufreinigung und Differenzierung von hES-Zellen }\end{array}$ \\
\hline Cellartis & Göteborg, Schweden & $\begin{array}{l}\text { Verfügt über ca. } 30 \text { eigene } h E S-Z e l l-L i n i e n \\
\text { Technologieentwicklung für drug development, Toxizitätstestung } \\
\text { Kooperation u. a. mit Pfizer (New York, USA) }\end{array}$ \\
\hline Cellular Dynamics & Madison, USA & $\begin{array}{l}\text { Gewinnung von kardialen Zellen aus hES-Zellen für Toxizitätstestung; Kooperation u. a. mit } \\
\text { Roche AG (Basel, Schweiz) }\end{array}$ \\
\hline ES Cell International & Singapur & $\begin{array}{l}\text { Bereitstellung von (teils GMP-konformen) hES-Zellen und abgeleiteten Zellen für Forschung } \\
\text { und drug discovery }\end{array}$ \\
\hline Geron & Menlo Park, USA & $\begin{array}{l}\text { Entwicklung von hES-Zell-basierten Therapien für mehrere Erkrankungen (Rückenmarkver- } \\
\text { letzungen, Morbus Parkinson, Diabetes, Herzerkrankungen u. a.) }\end{array}$ \\
\hline GlaxoSmithKline & London, GB & Forschungen zu drug discovery, sowohl eigene Forschungen als auch Kooperationen \\
\hline InvitroGen & Karlsbad, USA & $\begin{array}{l}\text { Entwicklung und Vertrieb von auf hES-Zellen basierenden Zell-Linien } \\
\text { Entwicklung und Vertrieb von research tools (Medien, Antikörper etc.) }\end{array}$ \\
\hline Millipore & Billerica, USA & $\begin{array}{l}\text { Vertrieb von aus } \mathrm{hES} \text {-Zellen abgeleiteten Zellen } \\
\text { Entwicklung und Vertrieb von research tools }\end{array}$ \\
\hline NovoCell & San Diego, USA & $\begin{array}{l}\text { Verfügt über mindestens } 6 \text { eigene hES-Zell-Linien (darunter eine GMP-konforme) } \\
\text { Entwicklung hES-Zell-basierter Therapien für Diabetes mellitus }\end{array}$ \\
\hline PlastiCell & London, GB & Entwicklung von Technologien für drug discovery \\
\hline Stem Cell Sciences & Edinburgh, GB & $\begin{array}{l}\text { Produktion spezifizierter Stammzellen aus } \mathrm{hES} \text {-Zellen zur Anwendung in der regenerativen } \\
\text { Medizin } \\
\text { Entwicklung von Medien etc. }\end{array}$ \\
\hline VistaGen & San Francisco, USA & Entwicklung von differenzierten Zell-Linien für drug discovery, Toxizitätstestung \\
\hline
\end{tabular}

taminierenden“ Fremdzellen, insbesondere undifferenzierte hES-Zellen, mehr enthält, die Ausgangspunkt für Tumorentstehung sein können. Um dieses Kriterium zu erfüllen, wurden in den letzten Jahren verschiedene Strategien entwickelt, die von der Etablierung hocheffektiver Aufreinigungsprotokolle für hES-Zell-abgeleitete Zellen über Methoden der Negativselektion (gegen undifferenzierte hESZellen) bis hin zur Entwicklung zytotoxischer Antikörper reichen, die spezifisch verbliebene hES-Zellen erkennen und eliminieren sollen (Übersicht in [57]).
2) Weiterhin besteht (wie generell bei der allogenen Zell- und Gewebetransplantation) das Problem der Immununverträglichkeit transplantierter Zellen, die als fremd erkannt und abgestoßen werden können. Dieses Problem könnte für hES-Zellen vermutlich durch die Bereitstellung einer großen Zahl von Zell-Linien mit unterschiedlicher Immunkompatibilität vermindert werden. So ist beispielsweise geschätzt worden, dass ca. 150 verschiedene hES-Zell-Linien ausreichend wären, um für $85 \%$ der britischen Bevölkerung immunkompatible Linien zur Verfügung zu haben
[58], wobei diese allerdings eine vergleichbare, universale Differenzierungsfähigkeit aufweisen müssten, was für viele Linien derzeit fraglich ist. Das Problem immunologischer Abwehrreaktionen könnte auch durch genetische Veränderungen an den hES-Zellen oder die Verkapselung der transplantierten Zellen in immunologisch kompatible Materialien gelöst werden. Gegebenenfalls könnten Immununverträglichkeiten auch durch Immunsuppression oder Induktion von Immuntoleranz vermindert werden. Auf diesen Gebieten werden international intensive Forschungsar- 
beiten durchgeführt (Übersicht in [59, $60])$.

Einige ausgewählte Beispiele für tierexperimentelle Studien sollen den derzeitigen Stand der präklinischen Forschung an hES-Zellen dokumentieren.

So ist es gelungen, aus hES-Zellen hoch aufgereinigte neurale Vorläuferzellen zu entwickeln, diese in dopaminerge Neurone zu differenzieren und erfolgreich in ein Ratten-Tiermodell mit Morbus Parkinson zu transplantieren. Die Zelltransplantation der dopaminergen Neurone führte zu deutlichen therapeutischen Effekten in den behandelten Tieren. 12 Wochen nach Transplantation wurden keinerlei Anzeichen von Tumorbildung beobachtet [61]. Weiterhin wurden neurale Vorläuferzellen, die aus hES-Zellen gewonnen wurden, in ein Rattenmodell des Schlaganfalls transplantiert, wo sie zu einer signifikanten Verbesserung der sensomotorischen Nervenfunktion beitrugen. Die transplantierten Zellen wanderten aus dem Injektionsgebiet in das geschädigte Gewebe, exprimierten neurale Differenzierungsmarker, und behandelte Tiere zeigten eine teilweise Wiederherstellung ihrer motorischen Leistungen. Auch hier wurden 8 Wochen nach Transplantation keine Teratome entdeckt [62].

Auch hinsichtlich der Herstellung und Transplantation von aus hES-Zell-abgeleiteten Kardiomyozyten wurden Fortschritte erzielt. Es wurde gezeigt, dass die Transplantation kardialer Vorläuferzellen in Nagermodelle für den Herzinfarkt zu einer signifikanten Besserung des klinischen Zustandes führte, und zwar nachweislich durch Bildung neuen Herzmuskelgewebes im Gebiet der Infarktnarbe. 6o Tage nach Transplantation konnte keine Teratom-Bildung beobachtet werden, wenn vordifferenzierte Zellen für die Transplantation verwendet wurden. Derzeitige Bemühungen sind weiterhin darauf gerichtet, transplantierbares vaskularisiertes Herzgewebe in vitro zu generieren $[63,64,65]$.

Im Rahmen der Bemühungen zur Herstellung funktioneller pankreatischer Beta-Zellen aus hES-Zellen wurde kürzlich ebenfalls ein wesentlicher Fortschritt erzielt. Es konnte gezeigt werden, dass sich aus hES-Zellen funktionsfähige, Glukose- sensitive Insulin-produzierende Zellen entwickelten, die nach Transplantation in Mäuse mit experimentell induziertem Diabetes den Blutglukosespiegel normalisierten. Die Entfernung des Transplantates führte wieder zum Anstieg des Blutzuckerspiegels. Da in dieser Studie noch kein Aufreinigungsschritt für die transplantierten Zellen eingeschlossen war, wurde in 1 von 46 untersuchten Transplantaten ein Tumor nachgewiesen [66].

Weitere tierexperimentelle Studien haben ebenfalls gezeigt, dass aus hES-Zellen abgeleitete spezialisierte Zellen auch für die Behandlung anderer als der im $\mathrm{Zu}$ sammenhang mit der Stammzellforschung häufig genannten degenerativen Erkrankungen ein großes Potenzial haben (Übersicht in [45]). Beispielsweise führte die Injektion von aus hES-Zellen abgeleiteten Blutgefäß-Vorläuferzellen in verschiedene Mausmodelle für Blutgefäßschädigungen (Extremitätenischämie, Herzinfarkt, diabetische Retinopathie) zur Gefäßneubildung im Bereich der jeweiligen Gefäßschädigung [67]. Die Behandlung mit aus hES-Zellen abgeleiteten Zellen des retinalen Pigmentepithels in einem Rattenmodell für genetisch bedingte Netzhautdegeneration ergab eine signifikante Verbesserung der visuellen Fähigkeiten der Tiere. Dies ist insofern von großer Bedeutung, da die Degeneration des retinalen Pigmentepithels in engem Zusammenhang mit der altersabhängigen Makuladegeneration steht, eine der Hauptursachen für Erblindung im Alter [68]. Bei all diesen genannten Studien handelt es sich zunächst um Proof-of-concept-Studien, die das therapeutische Potenzial von aus hESZellen hergestellten Zellen zunächst im Tiermodell belegen. Zweifelsohne sind weitere intensive Studien zur Verbesserung der Differenzierungs- und Aufreinigungsprotokolle sowie tierexperimentelle Studien - insbesondere Langzeitstudien und Studien unter Verwendung weiterer Versuchstiere - notwendig, bevor klinische Studien mit aus hES-Zellen abgeleiteten Zellen begonnen werden können.

Zusammenfassend soll nochmals darauf hingewiesen werden, dass Zellersatztherapien nur eines der möglichen Anwendungsgebiete von hES-Zellen sind und zudem einen längerfristigen zeit- lichen Horizont haben. Humane ES-Zellen haben derzeit vor allem ein großes Potenzial auch auf anderen Gebieten der medizinischen Forschung wie der Krankheitsursachen-Forschung oder der Pharmakologie und Toxikologie. Die Entdeckung der Reprogrammierbarkeit von somatischen Zellen zu induzierten pluripotenten Stammzellen (iPS-Zellen) hat darüber hinaus deutlich gemacht, dass die embryonale Stammzellforschung insgesamt auch die Aussicht auf ganz neue, unerwartete Erkenntnisse eröffnet, die zur Etablierung neuer Forschungsfelder führen können.

\section{Korrespondierender Autor}

\section{Professor Dr. Anna M. Wobus}

Leibniz-Institut für Pflanzengenetik und Kulturpflanzenforschung (IPK)

Corrensstraße 3

06466 Gatersleben, BRD

E-Mail: wobusam@ipk-gatersleben.de

\section{Literatur}

1. Thomson JA, Itskovitz-Eldor J, Shapiro SS, et al. (1998) Embryonic stem cell lines derived from human blastocysts. Sience 282:1145-1147

2. Löser P, Wobus MA (2007) Aktuelle Entwicklungen in der Forschung mit humanen embryonalen Stammzellen. Naturwiss Rundschau 60:229-241

3. Green RM (2007) Can we develop ethically universal embryonic stem-cell lines? Nat Rev Genet 8:480-485

4. Chung Y, Klimanskaya I, Becker S, et al. (2008) Human embryonic stem cell lines generated without embryo destruction. Cell Stem Cell 2:113-117

5. Peura TT, Bosman A, Stojanov T (2007) Derivation of human embryonic stem cell lines. Theriogenology 67:32-42

6. Peura T, Bosman A, Chami O, et al. (2008) Karyotypically normal and abnormal human embryonic stem cell lines derived from PGD-analyzed embryos. Cloning Stem Cells 10:Vorab-Publikation online

7. Munné S, Velilla E, Colls PM, et al. (2005) Selfcorrection of chromosomally abnormal embryos in culture and implications for stem cell production. Fertil Steril 84:1328-1334

8. Zhang X, Stojkovic P, Przyborski S, Cooke M (2006) Derivation of human embryonic stem cells from developing and arrested embryos. Stem Cells 24:2669-2676

9. Landry DW, Zucker HA (2004) Embryonic death and the creation of human embryonic stem cells. J Clin Invest 114:1184-1186

10. Ben-Yosef D, Malcov M, Eiges R (2008) PGDderived human embryonic stem cell lines as a powerful tool for the study of human genetic disorders. Mol Cell Endocrinol 282:153-158 
11. Eiges R, Urbach A, Malcov M, et al. (2007) Developmental study of fragile $X$ syndrome using human embryonic stem cells derived from preimplantation genetically diagnosed embryos. Cell Stem Cells 1:568-577

12. Ben-Nun IF, Benvenisty N (2006) Human embryonic stem cells as a cellular model for human disorders. Mol Cell Endocrinol 252:154-159

13. Zwaka TP, Thomson JA (2003) Homologous recombination in human embryonic stem cells. Nat Biotechnol 21:319-321

14. Wilmut I, Schnieke AE, McWhir J, et al. (1997) Viable offspring derived from fetal and adult mammalian cells. Nature 385:810-813

15. Lanza RP, Cibelli JB, West MD (1999) Human therapeutic cloning. Nat Med 5:975-977

16. French AJ, Adams CA, Anderson LS, et al. (2008) Development of human cloned blastocysts following somatic cell nuclear transfer with adult fibroblasts. Stem Cells 26:485-493

17. Hurlbut WB (2004) Altered nuclear transfer as a morally acceptable means for the procurement of human embryonic stem cells commissioned working paper. http://www.bioethics.gov/ background/hurlbut.html

18. Meissner A, Jaenisch R (2005) Generation of nuclear transfer-derived pluripotent $\mathrm{ES}$ cells from cloned Cdx2-deficient blastocysts. Nature 439: 212-215

19. Revazova ES, Turovets NA, Kochetkova OD, et al. (2007) Patient-specific stem cell lines derived from human parthenogenetic blastocysts. Cloning Stem Cells 9:432-449

20. Kim K, Ng K, Rugg-Gunn PJ, Shieh JH (2007) Recombination signatures distinguish embryonic stem cells derived by parthenogenesis and somatic cell nuclear transfer. Cell Stem Cell 1:346-352

21. Takahashi K, Yamanaka S (2006) Induction of pluripotent stem cells from mouse embryonic and adult fibroblast cultures by defined factors. Cell 126:663-676

22. Yu J, Vodyanik MA, Smuga-Otto K, et al. (2007) Induced pluripotent stem cell lines derived from human somatic cells. Science 318:1917-1920

23. Takahashi K, Tanabe K, Ohnuki M, et al. (2007) Induction of pluripotent stem cells from adult human fibroblasts by defined factors. Cell 131: 861-872

24. Wobus A (2008) Reversibilität des Entwicklungsstatus menschlicher Zellen. Naturwiss Rdsch 61: 221-225

25. Gebler B, Schöler H (2008) Durchbruch in der Stammzellforschung? Die Reprogrammierung von Körperzellen zu pluripotenten Stammzellen. Übersicht und Ausblick. Bundesgesundheitsbl Gesundheitsforsch Gesundheitsschutz 51:1005-1013

26. Adewumi O, Aflatoonian B, Ahrlund-Richter L, et al. (2007) Characterization of human embryonic stem cell lines by the International Stem Cell Initiative. Nat Biotechnol 25:803-816

27. Enver T, Soneji S, Joshi C, et al. (2005) Cellular differentiation hierarchies in normal and cultureadapted human embryonic stem cells. Hum Mol Gen 14:3129-3140

28. Silva SS, Rowntree RK, Mekhoubad S, Lee JT (2008) $\mathrm{X}$-chromosome inactivation and epigenetic fluidity in human embryonic stem cells. Proc Natl Acad Sci USA 105:4820-4825

29. Shen Y, Matsuno Y, Fouse SD, et al. (2008) X-inactivation in female human embryonic stem cells is in a nonrandom pattern and prone to epigenetic alterations. Proc Natl Acad Sci USA 105:4709-4714
30. Oh SK, Kim HS, Ahn HJ, et al. (2005) Derivation and Characterization of New Human Embryonic Stem Cell Lines: SNUhES1, SNUhES2, and SNUhES3. Stem Cells 23:211-219

31. Chang KH, Nelson AM, Cao H, et al. (2006) Definitive-like erythroid cells derived from human embryonic stem cells coexpress high levels of embryonic and fetal globins with little or no adult globin. Blood 108:1515-1523

32. Cowan CA, Klimanskaya I, McMahon J, et al. (2004) Derivation of embryonic stem-cell lines from human blastocysts. N Engl J Med 350:1353-1356

33. Osafune K, Caron L, Borowiak N, et al. (2008) Marked differences in differentiation propensity among human embryonic stem cell lines. Nat Biotechnol 26:313-315

34. Yamanaka S, Li J, Kania G, et al. (2008) Pluripotency of embryonic stem cells. Cell Tissue Res 331:5-22

35. Smith AG (2001) Embryo-derived stem cells: of mice and men. Annu Rev Cell Dev Biol 17: 4535-4562

36. Sato N, Meijer L, Skaltsounis L, et al. (2004) Maintenance of pluripotency in human and mouse embryonic stem cells through activation of Wnt signaling by a pharmacological GSK-3-specific inhibitor. Nat Med 10:55-63

37. Xu C, Rosler E, Jiang J, et al. (2005) Basic fibroblast growth factor supports undifferentiated human embryonic stem cell growth without conditioned medium. Stem Cells 23:315-323

38. Beattie GM, Lopez AD, Bucay N, et al. (2005) Activin a maintains pluripotency of human embryonic stem cells in the absence of feeder layers. Stem Cells 23:489-495

39. Brons IG, Smithers LE, Trotter MW, et al. (2007) Derivation of pluripotent epiblast stem cells from mammalian embryos. Nature 448:191-195

40. Wobus AM, Boheler KR (2005) Embryonic stem cells: prospects for developmental biology and cell therapy. Physiol Rev 85:635-678

41. Murry CE, Keller G (2008) Differentiation of embryonic stem cells to clinically relevant populations: lessons from embryonic development. Cell 132: 661-680

42. Skottman H, Narkilahti S, Hovatta O (2007) Challenges and approaches to the culture of pluripotent human embryonic stem cells. Regen Med 2:265-273

43. Ludwig TE, Levenstein ME, Jones JM, et al. (2006) Derivation of human embryonic stem cells in defined conditions. Nat Biotechnol 24:185-187

44. Crook JM, Peura TT, Kravets L, et al. (2007) The generation of six clinical-grade human embryonic stem cell lines. Cell Stem Cell 1:490-494

45. Deb KD, Sarda K (2008) Human embryonic stem cells: preclinical perspectives. J Translational Med $6: 7$

46. Franklin SB, Hunt C, Cornwell G, et al. (2008) hESCCO: development of good practice models for hES cell derivation. Regen Med 3:105-116

47. Améen C, Strehl R, Björquist P, et al. (2008) Human embryonic stem cells: current technologies and emerging industrial applications. Crit Rev Oncol Hematol 65:54-80

48. Rubin LL (2008) Stem cells and drug discovery: the beginning of a new era? Cell 132:549-552

49. Adler S, Pellizzer C, Hareng L, et al. (2008) First steps in establishing a developmental toxicity test method based on human embryonic stem cells. Toxicol in Vitro 22:200-211

50. Sartipy P, Björquist P, Strehl R, Hyllner J (2007) The application of human embryonic stem cell technologies to drug discovery. Drug Discov Today 12:688-699
51. Ek M, Söderdahl T, Küppers-Munther B, Edsbagge J (2007) Expression of drug metabolizing enzymes in hepatocyte-like cells derived from human embryonic stem cells. Biochem Pharmacol 74: 496-503

52. Hay DC, Zhao D, Ross A, Mandalam R, et al. (2007) Direct differentiation of human embryonic stem cells to hepatocyte-like cells exhibiting functional activities. Cloning Stem Cells 9:51-62

53. Agarwal S, Holton KL, Lanza R (2008) Efficient differentiation of functional hepatocytes from human embryonic stem cells. Stem Cells published online 21.2.2008

54. Welt online, 19.5.2008, http://www.welt.de/welt_ print/article2009292/Vorerst_keine_Therapie_ mit_embryonalen_Stammzellen_in_den_USA. html

55. Baker M (2008) FDA to vet embryonic stem cells' safety. Nature 452:670

56. Pressemitteilung von Advanced Cell Technology vom 1.2.2008

57. Hentze H, Graichen R, Colman A (2007) Cell therapy and the safety of embryonic stem cellderived grafts Trends. Biotechnol 25:24-32

58. Taylor CJ, Bolton EM, Pocock S, et al. (2005) Banking on human embryonic stem cells: estimating the number of donor cell lines needed for HLA matching. Lancet 366:2019-2025

59. Boyd AS, Higashi Y, Wood KJ (2005) Transplanting stem cells: potential targets for immune attack. Modulating the immune response against embryonic stem cell transplantation. Adv Drug Deliv Rev 57:1944-1969

60. Chidgey AP, Layton D, Trounson A, Boyd RL (2008) Tolerance strategies for stem-cell-based therapies. Nature 453:330-337

61. Cho MS, Lee YE, Kim JY, et al. (2008) Highly efficient and large-scale generation of functional dopamine neurons from human embryonic stem cells. PNAS 105:3392-3397

62. Daadi MM, Maag AL, Steinberg GK (2008) Adherent self-renewable human embryonic stem cellderived neural stem cell line: functional engraftment in experimental stroke model. PLoS One, 3, E1644

63. Caspi O, Huber I, Kehat I, et al. (2007). Transplantation of human embryonic stem cell-derived cardiomyocytes improves myocardial performance in infarcted rat hearts. J Am Coll Cardiol 50: 1884-1893

64. Caspi O, Lesman A, Basevitch Y, et al. (2007) Tissue engineering of vascularized cardiac muscle from human embryonic stem cells. Circ Res 100: 263-272

65. Laflamme MA, Chen KY, Naumova AV, et al. (2007) Cardiomyocytes derived from human embryonic stem cells in pro-survival factors enhance function of infarcted rat hearts. Nat Biotechnol 25: 1015-1024

66. Kroon E, Martinson LA, Kadoya K, et al. (2008) Pancreatic endoderm derived from human embryonic stem cells generates glucose-responsive insulinsecreting cells in vivo. Nat Biotechnol 26:443-452

67. Lu SJ, Feng Q, Caballero S, Chen Y (2007) Generation of functional hemangioblasts from human embryonic stem cells. Nat Methods 4:501-509

68. Lu B, Wang S, Girman S, et al. (2007) GMP-compliant Human RPE cells derived from embryonic stem cell Lines rescue visual function in a rat model for photoreceptor degeneration. Poster. http://www. advancedcell.com/file_download/225 